\title{
Cost-effectiveness of $2+1$ dosing of 13-valent and 10-valent pneumococcal conjugate vaccines in Canada
}

\author{
Stephanie R Earnshaw ${ }^{*}$, Cheryl L McDade ${ }^{1}$, Giovanni Zanotti ${ }^{2}$, Raymond A Farkouh ${ }^{3}$ and David Strutton ${ }^{3}$
}

\begin{abstract}
Background: Thirteen-valent pneumococcal conjugate vaccine (PCV13) and 10-valent pneumococcal conjugate vaccine (PCV10) are two recently approved vaccines for the active immunization against Streptococcus pneumoniae causing invasive pneumococcal disease in infants and children. PCV13 offers broader protection against Streptococcus pneumoniae; however, PCV10 offers potential protection against non-typeable Haemophilus influenza (NTHi). We examined public health and economic impacts of a PCV10 and PCV13 pediatric national immunization programs (NIPS) in Canada.
\end{abstract}

Methods: A decision-analytic model was developed to examine the costs and outcomes associated with PCV10 and PCV13 pediatric NIPs. The model followed individuals over the remainder of their lifetime. Recent disease incidence, serotype coverage, population data, percent vaccinated, costs, and utilities were obtained from the published literature. Direct and indirect effects were derived from 7-valent pneumococcal vaccine. Additional direct effect of $4 \%$ was attributed to PCV10 for moderate to severe acute otitis media to account for potential NTHi benefit. Annual number of disease cases and costs (2010 Canadian dollars) were presented.

Results: In Canada, PCV13 was estimated to prevent more cases of disease (49,340 when considering both direct and indirect effects and 7,466 when considering direct effects only) than PCV10. This translated to population gains of 258 to 13,828 more quality-adjusted life-years when vaccinating with PCV13 versus PCV10. Annual direct medical costs (including the cost of vaccination) were estimated to be reduced by $\$ 5.7$ million to $\$ 132.8$ million when vaccinating with PCV13. Thus, PCV13 dominated PCV10, and sensitivity analyses showed PCV13 to always be dominant or cost-effective versus PCV10.

Conclusions: Considering the epidemiology of pneumococcal disease in Canada, PCV13 is shown to be a cost-saving immunization program because it provides substantial public health and economic benefits relative to PCV10.

Keywords: Vaccine, Cost-effectiveness, Pneumococcal conjugate vaccine, Pneumococcal disease

\section{Background}

A 7-valent pneumococcal polysaccharide-protein conjugate vaccine, Prevenar (PCV7), licensed for use in Canada in 2001 in children, has led to a dramatic decrease in Streptococcus pneumoniae-related diseases such as acute otitis media (AOM), pneumonia (PNE), and invasive pneumococcal disease (IPD) in Canada [1-8]. Two pneumococcal conjugate vaccines have recently been approved for use in infants in Canada. The 10-valent

\footnotetext{
*Correspondence: searnshaw@rti.org

${ }^{1}$ RTI Health Solutions, 200 Park Offices Drive, Research Triangle Park, NC 27709, USA

Full list of author information is available at the end of the article
}

pneumococcal conjugate vaccine (PCV10) [9] offers seroprotection against the original seven pneumococcal serotypes plus three additional serotypes 1,5 , and 7F. The 10 serotypes are conjugated to three different proteins, one of which is protein $\mathrm{D}$, which may provide protection against non-typeable Haemophilus influenzae (NTHi) [10]. The 13-valent pneumococcal conjugate vaccine Prevenar 13 (PCV13) provides the same seroprotection as PCV7 [11] and contains the 10 serotypes in PCV10 plus three additional pneumococcal serotypes: $3,6 \mathrm{~A}$, and 19A [11]. PCV10 was originally approved with a $3+1$ dosing schedule but was recently approved for a $2+1$ dosing
Ciomed Central

(c) 2012 Earnshaw et al.; licensee BioMed Central Ltd. This is an Open Access article distributed under the terms of the Creative Commons Attribution License (http://creativecommons.org/licenses/by/2.0), which permits unrestricted use, distribution, and reproduction in any medium, provided the original work is properly cited. 
schedule in Europe [12]; it previously was used in Quebec with a $2+1$ schedule [9]. As of January 2011, after Ontario and Quebec switched from PCV10 to PCV13, all provinces in Canada currently are using PCV13 in their immunization programs. Understanding the value for money or cost-effectiveness of vaccine policies with these newer vaccines is important.

Recently published analyses have examined the cost-effectiveness of PCV10 compared with PCV7 in Canada [13]. Analyses comparing the use of PCV13 with PCV10 in the provinces of Alberta [14] and Quebec [15,16] also have been performed. Although Chuck et al. and Roussy et al. examined the value of PCV10 with a $2+1$ dosing schedule, the Canadian-specific analysis by Talbird et al. examined the use of PCV10 with a $3+1$ dosing schedule. In the current analysis, we expanded on previously published analyses by comparing costs and outcomes associated with the use of PCV13 compared with PCV10 using a two-dose primary series followed by a booster dose at 12 to 15 months across all provinces and territories in Canada.

\section{Methods}

\section{Model design}

A decision-analytic model (Figure 1) was constructed to examine the costs and outcomes of vaccine policies that included PCV13 and PCV10 for vaccinating children against Streptococcus pneumoniae, which causes IPD (bacteremia and meningitis), PNE (hospitalized and ambulatory), and AOM (mild and moderate/severe). The model was programmed in Microsoft Excel version 2003.

Individuals entered the model either vaccinated or not vaccinated, depending upon their ages and vaccination uptake. Individuals were followed over the remainder of their lifetime, in which they were at risk for contracting IPD, PNE, or AOM based on their vaccination status. If individuals contracted IPD, they had a risk of developing sequelae such as hearing loss and/or neurological impairment or dying from IPD. Individuals contracting PNE had a risk of dying if they were hospitalized. Children who contracted moderate/severe AOM were at risk for requiring myringotomy.

Cases of disease were derived from population, epidemiology, and clinical data from the published literature. Specific data included Canadian populations, incidence of disease, serotype coverage, and direct and indirect effects associated with each vaccine. Based on the estimated disease cases, mortality and disease sequelae were estimated. Costs and utilities from the published literature were applied to derive the overall costs and health benefits for individuals on each vaccine. Costs and outcomes were presented from a Canadian health system perspective. Costs were reported in 2010 Canadian dollars.

\section{Population}

The model examined the impact of implementing pediatric vaccine policies, including PCV13 and PCV10,

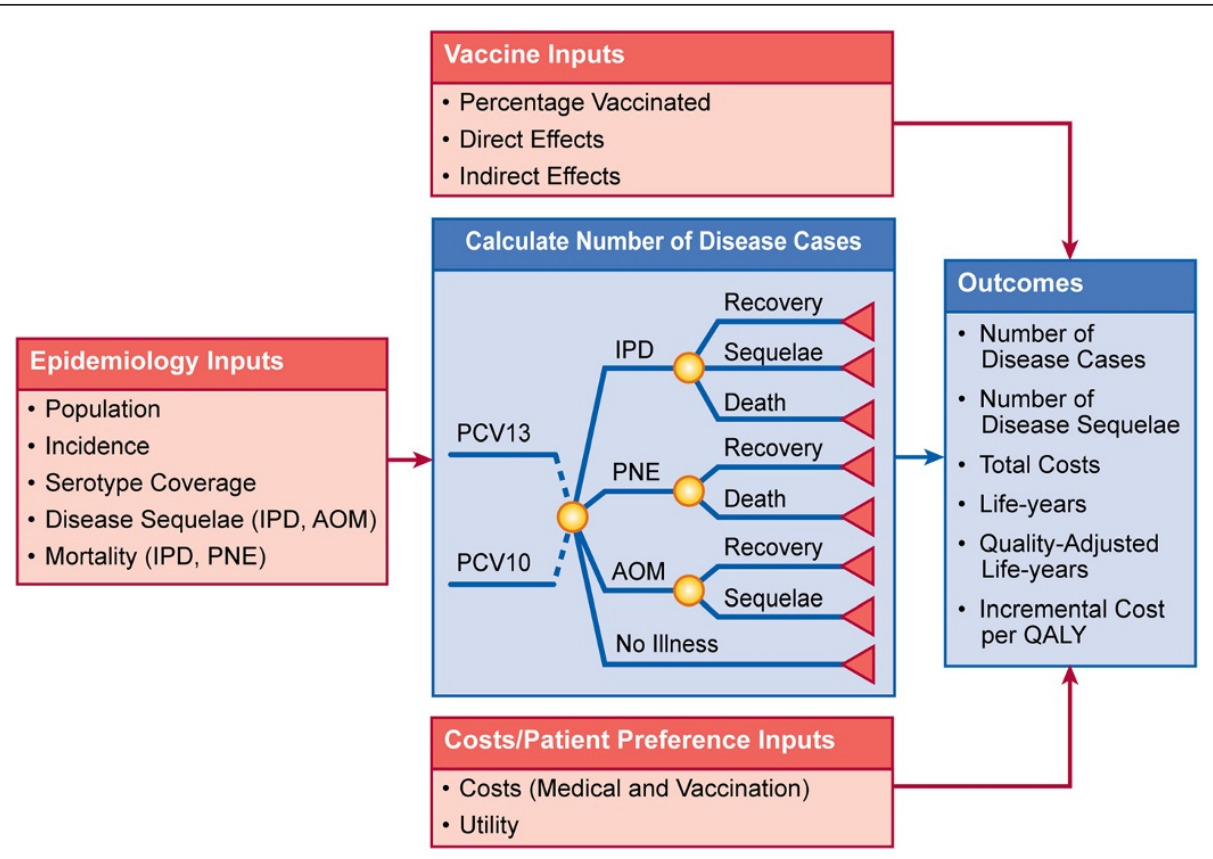

Figure 1 Model structure. $A O M=$ acute otitis media; $I P D=$ invasive pneumococcal disease; $P C V 10=10$-valent pneumococcal conjugate vaccine; PCV13 = 13-valent pneumococcal conjugate vaccine; PNE = pneumonia; QALY= quality-adjusted life-year. 
among individuals of all ages in Canada. Specifically, 34,108,000 individuals within Canada were considered [17]; of these, $1.4 \%$ or 477,512 children $<2$ years of age were considered for the vaccinated cohort. In the base case, we assumed $91 \%$ of these children would be vaccinated [2]. Vaccination uptake was examined in sensitivity analysis, where an uptake of $87 \%$ was examined [18]. The remaining individuals comprised the non-vaccinated cohorts, which included individuals aged 2 to $<5,5$ to $<18,18$ to $<65$, and $65+$ years.

\section{Inputs}

Model inputs are categorized as epidemiological, vaccinerelated, and economic inputs. Each is discussed separately.

\section{Epidemiology \\ Disease incidence}

As a result of the introduction of PCV7, a dramatic decrease in the incidence of PCV7-typed disease has occurred over time $[2,18,23,24]$. Thus, time since the introduction of PCV7 is a significant influencer of incidence in a population. Because of the limited availability of data and the need for use of the most current data, the incidence of IPD for Canada was estimated from the "Programme de surveillance du pneumocoque du Quebec" [18] From this report, the number of IPD cases by age were comprised of $88 \%$ bacteremia (isolated from blood) and $3.1 \%$ meningitis (isolated from cerebrospinal fluid) [18], consistent with previously published cost-effectiveness research [15]. Incidence of IPD from Morrow et al. [19] was examined in sensitivity analysis as the Morrow et al. [19] data are all-Canada specific.

Incidence of PNE was estimated from Morrow et al., Petit et al., and Nelson et al. [19-21]. Specifically, Nelson et al. [21] examined the impact of introducing a 7-valent pneumococcal conjugate vaccine for children and adults in the United States. Within this study, the authors calculated incidence rate ratios comparing a period of time after (2003-2004) with a period before (1998-2000) the introduction of the national immunization program. Using these incidence rate ratios for outpatient and hospitalized PNE, the incidence of pneumococcal PNE as reported by Morrow et al. [19] for Canada was adjusted. All-cause PNE then was estimated by multiplying by the ratio of pneumococcal PNE to all-cause PNE as reported by Petit et al. [20].

Incidence of AOM was estimated from the number of AOM claims reported in Quebec in 2007 [22]. In this study, the authors reported 44 claims per 100 personyears, of which $13.5 \%$ were seen by an ear, nose, and throat specialist [22]. The model assumed ear, nose, and throat specialist claims as moderate/severe AOM and all other claims as mild AOM. Incidence was further distributed by age based on the monthly frequency of physician claims by age [22]. Incidence of AOM from Morrow et al. [19] was examined in sensitivity analysis. Disease incidence by age is presented in Table 1 .

\section{Serotype coverage}

Serotype coverage for IPD for PCV10 and PCV13 were obtained from the National Centre for Streptococcus, from which isolates from all Canadian territories (excluding Quebec) were collected over the years [23]. Vaccinespecific serotype coverage was obtained by age for 2009 , the most current available year. These data are presented in Table 1. We did not consider cross-protection to $6 \mathrm{~A}$ or 19A for PCV10 because there was limited evidence of vaccine effectiveness against either potentially cross-reactive serotype.

Sensitivity analyses were performed using serotype coverage obtained from the Quebec surveillance program [18] from 2009 and the Calgary Area Streptococcus pneumoniae Epidemiology Research group from 2007 [2]. Sensitivity analyses also were performed with serotype coverage estimated by Bettinger et al., in which isolates from 12 centers of the Canadian Immunization Monitoring Program ACTive were examined [24,25]. These data also were used within the analyses performed by Talbird et al. [25]. All sensitivity analysis on serotype coverage data are presented in Additional file 1: Table A1. We also examined the impact of increasing serotype coverage of PCV10 relative to PCV13 to account for uncertainty in the serotype coverage data.

PNE and AOM were considered from an all-cause perspective because etiology is typically not determined for cases of mucosal disease. Thus, serotype coverage data were implicitly considered within vaccine effectiveness.

\section{Mortality}

General mortality in individuals was assumed to be similar to that observed in the general Canadian population [26]. IPD-specific and inpatient PNE mortality was obtained from Scheifele et al. [27] and Jette et al. [28] and is presented in Table 1. Deaths due to outpatient $\mathrm{PNE}$ and $\mathrm{AOM}$ were assumed to not occur.

\section{Direct effects of vaccines}

Direct effects are the direct reductions in disease cases that occur in individuals due to the individual being vaccinated. These effects are specific to the covered serotypes for IPD and are all-cause for PNE and AOM. As of the writing of this article, efficacy and effectiveness data for PCV13 and PCV10 were not available. However, interim analyses of surveillance data and efficacy trials have been presented $[29,30]$, and full data and details are expected to be available in the future. In the absence of such data, the effects for these vaccines were derived from the direct effects observed by individuals 
Table 1 Input parameters

\begin{tabular}{|c|c|c|c|c|c|c|}
\hline \multirow[t]{2}{*}{ Parameters } & \multicolumn{5}{|c|}{ Age (Years) } & \multirow{2}{*}{$\begin{array}{l}\text { Source(s)/ } \\
\text { Assumptior }\end{array}$} \\
\hline & $0-2$ & $2-4$ & $5-17$ & $18-64$ & $65+$ & \\
\hline \multicolumn{7}{|l|}{ Incidence (per 100,000) } \\
\hline Bacteremia & 14.77 & 10.62 & 6.74 & 43.67 & 87.07 & [18] \\
\hline Meningitis & 0.52 & 0.37 & 0.24 & 1.54 & 3.07 & \\
\hline Inpatient PNE & $1,135.13$ & 380.99 & 170.84 & 102.76 & $1,343.40$ & {$[19-21]$} \\
\hline Outpatient PNE & 874.77 & 460.69 & 514.63 & 55.35 & 63.31 & \\
\hline Mild AOM & $13,762.21$ & $13,811.12$ & $10,486.67$ & 0.00 & 0.00 & {$[22]$} \\
\hline Moderate/severe AOM & $2,147.86$ & $2,155.49$ & $1,636.65$ & 0.00 & 0.00 & \\
\hline \multicolumn{7}{|l|}{ Serotype coverage } \\
\hline PCV10 & 0.18 & 0.23 & 0.26 & 0.28 & 0.22 & [23] \\
\hline PCV13 & 0.61 & 0.68 & 0.42 & 0.55 & 0.55 & \\
\hline \multicolumn{7}{|l|}{ Mortality } \\
\hline Bacteremia & 0.01 & 0.003 & 0.003 & 0.07 & 0.11 & {$[26-28]$} \\
\hline Meningitis & 0.02 & 0.003 & 0.003 & 0.07 & 0.11 & \\
\hline Inpatient PNE & 0.01 & 0.003 & 0.003 & 0.07 & 0.11 & \\
\hline \multicolumn{7}{|l|}{ Direct medical costs } \\
\hline Bacteremia & $\$ 10,578.71$ & $\$ 3,379.85$ & $\$ 9,768.95$ & $\$ 13,559.64$ & $\$ 11,913.26$ & {$[19,51]$} \\
\hline Meningitis & $\$ 48,382.04$ & $\$ 24,615.09$ & $\$ 61,114.72$ & $\$ 22,409.11$ & $\$ 11,782.22$ & \\
\hline Inpatient PNE & $\$ 3,450.13$ & $\$ 2,149.73$ & $\$ 10,305.00$ & $\$ 9,606.02$ & $\$ 8,370.61$ & \\
\hline Outpatient PNE & $\$ 138.18$ & $\$ 69.09$ & $\$ 184.24$ & $\$ 126.66$ & $\$ 103.63$ & \\
\hline Mild AOM & $\$ 95.30$ & $\$ 47.65$ & $\$ 71.47$ & $\$ 0.00$ & $\$ 0.00$ & \\
\hline Moderate/severe AOM & $\$ 95.30$ & $\$ 47.65$ & $\$ 71.47$ & $\$ 0.00$ & $\$ 0.00$ & \\
\hline Utilities & 0.97 & 0.97 & 0.97 & 0.88 & 0.82 & [54] \\
\hline
\end{tabular}

AOM acute otitis media; PCV10 10-valent pneumococcal conjugate vaccine; $P C V 13$ 13-valent pneumococcal conjugate vaccine; $P N E$ pneumonia.

vaccinated with PCV7, a similar approach utilized by previous analyses $[13,14,25,31,32]$. Specific direct effects for each vaccine in the different diseases are discussed next.

Direct effects for PCV7 for preventing IPD among covered serotypes was reported to be 0.94 by Black et al. [33] We assumed that direct effects on covered serotypes experienced by individuals vaccinated with PCV10 or PCV13 would be similar to those experienced by individuals vaccinated with PCV7. Deceuninck et al. [34] performed a study of children receiving PCV7 in a $2+1$ dosing schedule in Quebec; the authors reported an effectiveness of 0.92 on IPD cases. We examined the effects of this reduced effectiveness in sensitivity analysis.

When using the Percentages of Children Achieving the Serotype-Specific Antibody Threshold of $0.2 \mu \mathrm{g} / \mathrm{mL}$, as Measured 1 Month After Completion of the Primary Series as a criteria for comparison (i.e., criteria defined by World Health Organization) the immunogenicity data for PCV10 was non-inferior to PCV7 for serotypes $4,9 \mathrm{~V}, 14$, $18 \mathrm{C}$, and $19 \mathrm{~F}$ [35], However, other response measures of PCV10 against common serotypes with PCV7 such as geometric mean concentrations of serotype-specific antibodies, as measured 1 month after completion of the primary series indicated a potentially lower response to PCV10 compared with PCV7. As a result, we examined the potential effect of lower immunogenic response in sensitivity analysis, in which direct effects for IPD, inpatient PNE, outpatient PNE, and all AOM were reduced to $0.90,0.85,0.80$, and 0.75 of the serotype extrapolated direct effects, respectively. Larger reductions in direct effects for PNE and AOM than for IPD were assumed because attaining seroprotection against mucosal disease was more difficult than against IPD [36]. Lower immunogenic response for PCV13 was not assumed because PCV13 shares the same carrier protein as PCV7 and has a comparable immunogenic response [37-39].

Direct effects in preventing PNE and AOM were obtained for all-cause PNE and all-cause AOM. Direct effects of PCV7 in preventing inpatient PNE was estimated at 0.26 [40]. Outpatient PNE was estimated at 0.06 [41]. Although PCV10 and PCV13 covered more serotypes than $\mathrm{PCV} 7$, the serotype coverage of $\mathrm{PCV} 7$ when it was introduced was greater than the potential current serotype coverage of both PCV13 and PCV10. Therefore, the direct effects for PCV10 and PCV13 were assumed to be similar to PCV7 but then adjusted downward based on PCV7's coverage prior to PCV7 
introduction relative to PCV10's or PCV13's current serotype coverage. Thus, PCV13 was assumed to have 0.19 and 0.04 direct effects for inpatient and outpatient PNE, respectively. PCV10 was assumed to have 0.05 and 0.01 direct effects for inpatient and outpatient PNE respectively.

Direct effects of PCV7 in preventing mild AOM and moderate/severe AOM was estimated at 0.07 [40] and 0.15 [42], respectively. Similar to PNE, direct effects for PCV10 and PCV13 were derived by adjusting the PCV7 direct effects by serotype coverage pre-introduction of PCV7 relative to PCV10's or PCV13's current serotype coverage. Thus, PCV13 was assumed to have 0.05 and 0.11 direct effects for mild and moderate/severe AOM, respectively. PCV10 was assumed to have 0.01 and 0.03 direct effects for mild and moderate/severe AOM, respectively. The direct effects for PCV10 in moderate/severe $\mathrm{AOM}$ were further increased to account for potential coverage of NTHi. Specifically, in the POET trial [10], perprotocol vaccine efficacy against NTHi AOM was reported as $31.1 \%$. The etiology of NTHi in the control group was 63 NTHi cases out of 499 AOM cases. Therefore, the reduction in AOM due to NTHi was estimated as an absolute increase in effectiveness of $0.04(31.1 \% \times 63 / 499)$. It was assumed that AOM etiology in POET would be similar to that observed in the moderate/severe cases in Canada.

\section{Indirect effects}

Indirect effects, or herd protection, occur when an unvaccinated individual does not contract pneumococcal disease because other individuals who have been vaccinated are less likely to carry vaccine-type pneumococci [43]. Robust indirect effects have been demonstrated for PCV7 national immunization programs where the seven serotypes in PCV7 have been reduced dramatically [44-47]. Indirect effects for PCV7 are presented in Table 2. Indirect effects for PCV13 were assumed to occur because PCV13 shares the same carrier protein as PCV7, has demonstrated a similar immune response to PCV7 [37-39], and has shown a statistically significant reduction in nasopharyngeal carriage [48]. In addition, a recent study by the Centers for Disease Control (CDC) has demonstrated that PCV13-type IPD has declined in adults (i.e., nonvaccinated individuals) in the US [49]. As a result, similar to estimating direct effects for PNE and

Table 2 Indirect effects, immunogenicity, and indirect effect adjustments for PCV7, PCV10, and PCV13

\begin{tabular}{|c|c|c|c|c|c|c|c|}
\hline & Bacteremia & Meningitis & $\begin{array}{l}\text { Inpatient } \\
\text { PNE }\end{array}$ & $\begin{array}{l}\text { Outpatient } \\
\text { PNE }\end{array}$ & $\begin{array}{l}\text { Mild } \\
\text { AOM }\end{array}$ & $\begin{array}{l}\text { Moderate/Severe } \\
\text { AOM }\end{array}$ & $\begin{array}{l}\text { Source(s)/ } \\
\text { Assumption }\end{array}$ \\
\hline \multicolumn{8}{|l|}{ Indirect effects (years) } \\
\hline \multicolumn{8}{|l|}{ PCV7 } \\
\hline $0-2$ & 0.68 & 0.68 & 0.07 & 0.16 & 0.21 & 0.15 & {$[40,42,44,65]$} \\
\hline $3-4$ & 0.68 & 0.68 & 0.00 & 0.00 & 0.00 & 0.00 & \\
\hline $5-17$ & 0.39 & 0.39 & 0.00 & 0.00 & 0.00 & 0.00 & \\
\hline $18-64$ & 0.47 & 0.47 & 0.26 & 0.00 & 0.00 & 0.00 & \\
\hline $64+$ & 0.36 & 0.36 & 0.00 & 0.00 & 0.00 & 0.00 & \\
\hline \multicolumn{8}{|c|}{ PCV10 (for Sensitivity analysis), } \\
\hline $0-2$ & 0.41 & 0.41 & 0.04 & 0.10 & 0.13 & 0.09 & \\
\hline $3-4$ & 0.43 & 0.43 & 0.00 & 0.00 & 0.00 & 0.00 & \\
\hline $5-17$ & 0.26 & 0.26 & 0.00 & 0.00 & 0.00 & 0.00 & \\
\hline $18-64$ & 0.39 & 0.39 & 0.22 & 0.00 & 0.00 & 0.00 & \\
\hline $64+$ & 0.28 & 0.28 & 0.00 & 0.00 & 0.00 & 0.00 & \\
\hline \multicolumn{8}{|l|}{ PCV13 } \\
\hline $0-2$ & 0.59 & 0.59 & 0.06 & 0.14 & 0.18 & 0.13 & \\
\hline $3-4$ & 0.62 & 0.62 & 0.00 & 0.00 & 0.00 & 0.00 & \\
\hline $5-17$ & 0.30 & 0.30 & 0.00 & 0.00 & 0.00 & 0.00 & \\
\hline $18-64$ & 0.55 & 0.55 & 0.30 & 0.00 & 0.00 & 0.00 & \\
\hline $64+$ & 0.42 & 0.42 & 0.00 & 0.00 & 0.00 & 0.00 & \\
\hline $\begin{array}{l}\text { Immuno-genicity for } \\
\text { PCV10 Direct Effects }\end{array}$ & 0.90 & 0.90 & 0.85 & 0.80 & 0.75 & 0.75 & {$[36]$} \\
\hline
\end{tabular}

AOM acute otitis media; $P C V 7$ 7-valent pneumococcal polysaccharide-protein conjugate vaccine; $P C V 10$ 10-valent pneumococcal conjugate vaccine;

${ }^{b}$ Indirect effects for PCV10 are assumed to be 0 in the base case. 
AOM, indirect effects for PCV13 were derived by adjusting the PCV7 indirect effects by serotype coverage preintroduction of PCV7 relative to PCV13 current serotype coverage. Indirect effects in the presence of PCV10 were assumed to not occur in the base-case analysis because the 11-valent investigational vaccine that preceded PCV10 and PCV10 did not show a consistent statistically significant effect on pneumococcal nasopharyngeal carriage $[10,50,51]$. Indirect effects for PCV10 were examined in sensitivity analyses. Indirect effects for PCV10 in these analyses were assumed to be similar to estimating direct effects for PNE and AOM, in that indirect effects for PCV10 were derived by adjusting the PCV7 indirect effects by serotype coverage pre-introduction of $\mathrm{PCV} 7$ relative to PCV10 current serotype coverage. These data are presented in Table 2.

\section{Economic inputs}

\section{Costs}

The model considered direct and indirect medical costs necessary to treat each case of disease, each myringotomy, and any vaccine-acquisition costs. Direct medical costs were assumed to include diagnostics, physician time, hospitalization, prescriptions, and over-the counter medications as needed. These costs (Table 1) were obtained from Morrow et al. [19] and inflated to 2010 values using the Canadian-specific consumer price index multiplier [52].

Vaccination costs were based on the acquisition cost per dose plus a direct administration fee. List price acquisition cost per dose was assumed to be $\$ 86.26$ for PCV13. Because acquisition cost of PCV10 was not available as of the writing of this article, the cost was assumed to be similar to the cost of PCV13. Administration cost per dose was estimated as $\$ 7.84$ [52,53]. The impact of vaccine costs was examined in sensitivity analysis; a breakeven analysis is presented.

\section{Utilities}

Utility weights allow for an objective measurement of the desirability of a health state in a cost-utility analysis. A utility of 1.0 represents perfect health, whereas a value of 0.0 represents death. When combined with life-years, utilities produce quality-adjusted life-years (QALYs).

General health utility was obtained for the general Canadian population from the Canadian Community Health Survey [54]. This general health utility was calculated as weighted average of portion of individuals in each age group, and utility values reported. General health utilities for each age group are presented in Table 1.

Utility decrements due to neurological impairment and hearing loss were obtained from Morrow et al. [19] and were assumed to be 0.6 and 0.8 , respectively.
These decrements were similar to those used in previous Canadian specific analyses $[13,14]$.

\section{Model calculations}

The model presented the following annual outcomes: number of disease cases, sequelae, deaths, and costs. In addition, population life-years and QALYs were calculated along with incremental cost per QALY, which was calculated as the difference in total costs between PCV13 and PCV10 divided by the difference in QALYs in populations in which a PCV10 versus a PCV13 vaccination policy was implemented. If a strategy was less costly and more effective, it was a dominant strategy, referred to as cost-saving.

Several sensitivity and scenario analyses were performed. Specifically, we examined the impact of the following parameters on results: reduction in the percentage of children vaccinated, reduced immunogenicity of PCV10 direct effects, full indirect effects for PCV10, indirect effects for PCV10 at 50\% of indirect effects that might occur, combined reduced immunogenicity of PCV10 direct effects and indirect effects at 50\% of expected occurrence, combined reduced immunogenicity of PCV10 direct effects and indirect effects at 100\% of expected occurrence, no direct effects on NTHi for PCV10, PCV13 indirect effects at 50\% of indirect effects that might occur, increase in serotype coverage for PCV10 when accounting for cross-reactively with 6A, incidence of IPD and AOM as reported by Morrow et al. [19], serotype coverage as reported by Institut National de Sante Publique du Quebec [18], serotype coverage as reported for Alberta by Kellner et al. [2], direct effects for IPD as reported for a $2+1$ dosing by Deceuninck et al. [34], 3+1 dosing schedule for PCV10, inclusion of indirect medical costs, and mortality as reported in Chuck et al. [14]. Results are presented in figure format.

\section{Results}

\section{Base-case analysis}

Table 3 presents the annual number of disease cases that would occur in Canada under each vaccination policy when considering only direct effects and direct and indirect effects.

We observed 49,340 more cases of disease were prevented with PCV13 when both direct and indirect effects were considered. Specifically, 4,084 cases of bacteremia, 144 of meningitis, 8,760 of inpatient PNE, 6,201 of outpatient PNE, 27,001 of mild AOM, and 3,150 of moderate/ severe AOM were prevented annually when vaccinating children with PCV13. This translated to 49 more myringotomies and 29 disease sequelae being prevented annually when both direct and indirect effects were considered. As a result, the population gained 15,283 more life-years and 13,828 more QALYs when vaccinating children with 
Table 3 Annual number of disease cases in canada under each vaccine program

\begin{tabular}{|c|c|c|c|c|c|c|}
\hline \multirow[t]{2}{*}{ Program } & \multicolumn{2}{|l|}{ IPD } & \multicolumn{2}{|l|}{ PNE } & \multicolumn{2}{|l|}{ AOM } \\
\hline & Bacteremia & Meningitis & In-patient & Out-patient & Mild & Moderate/Severe \\
\hline \multicolumn{7}{|c|}{ Direct and indirect effects } \\
\hline PCV13 & 10,427 & 368 & 101,325 & 257,356 & 762,343 & 118,999 \\
\hline PCV10 & 14,511 & 512 & 110,085 & 263,557 & 789,344 & 122,150 \\
\hline \multicolumn{7}{|c|}{ Direct effects only } \\
\hline PCV13 & 14,459 & 510 & 108,785 & 262,485 & 785,018 & 121,437 \\
\hline PCV10 & 14,511 & 512 & 110,085 & 263,557 & 789,344 & 122,150 \\
\hline
\end{tabular}

AOM acute otitis media; IPD invasive pneumococcal disease; $P C V 10$ 10-valent pneumococcal conjugate vaccine; $P C V 13$ 13-valent pneumococcal conjugate vaccine; PNE pneumonia.

PCV13 versus PCV10. Approximately 879 deaths could be prevented annually and annual direct medical costs were reduced by $\$ 132.8$ million when vaccinating children with PCV13 versus than PCV10. Thus, PCV13 was found to dominate PCV10. Results are presented in Table 4.

When considering direct effects only, we observed 7,466 more cases of disease (52 more cases of bacteremia, 2 of meningitis, 1,300 of inpatient PNE, 1,072 of outpatient PNE, 4,327 of mild AOM, and 713 of moderate/severe AOM) were prevented annually when vaccinating children with PCV13 versus PCV10. This translated to 11 more myringotomies and 0.3 more disease sequelae of neurological impairment and hearing loss prevented annually. As a result, the population gained 287 more life-years and 258 more QALYs when vaccinating children with PCV13 versus PCV10. Approximately 14 deaths could be prevented annually and annual direct medical costs (including the cost of vaccination) were reduced by $\$ 5.7$ million when vaccinating children with PCV13 versus than PCV10. Thus, PCV13 was found to dominate PCV10. Results are presented in Table 5.

Assuming a total per-dose cost of PCV10 of $\$ 94.10$ (acquisition and administration) and considering a threshold incremental cost per QALY of $\$ 50,000$, the cost per dose for PCV13 could be as high as $\$ 737.11$ to remain cost-effective, when both direct and indirect effects were considered. PCV13 is cost neutral at a cost per dose of $\$ 197.72$ or at a $110 \%$ increase in cost per dose. The percentages of allowable price increases in PCV13 vaccine over PCV10 vaccine in order to maintain various cost-effectiveness levels is presented in Figure 2. We also examined the effect of reducing the cost per dose of PCV10 while maintaining a cost per dose of $\$ 94.10$ for PCV13 and found that even if the cost per dose of PCV10 were $\$ 0$, cost neutrality still could not be achieved (data no shown).

\section{Sensitivity analyses}

Overall, in all scenarios of sensitivity analysis, PCV13 prevented more cases of disease and remained cost-saving compared with PCV10 (Figure 3). Using serotype coverage as reported by Bettinger et al. [24] showed PCV13 to gain the highest total QALYs $(17,956)$, whereas assuming indirect effect for PCV10 similar to that seen for PCV7 showed the lowest total gain in QALYs $(5,102)$. In these scenarios, the greatest $(\$ 174,312,074)$ and the lowest $(\$ 62,824,747)$ reduction in total annual costs were observed, respectively. Changes in overall QALYs gained and annual costs saved for all other scenario fell between these ranges.

Table 4 Base-case cost and outcomes when considering both direct and indirect effects

\begin{tabular}{llll}
\hline Outcome & PCV13 & PCV10 & Difference(PCV13 - PCV10) \\
\hline Total life-years & $605,602,153$ & $605,586,870$ & 15,283 \\
Total QALYs & $543,278,132$ & $543,264,303$ & 13,828 \\
\hline Annual number of cases & 1,868 & & -49.5 \\
Myringotomy & 25.7 & 1,918 & -10.1 \\
Neurological impairment & 47.8 & 35. & -18.7 \\
Hearing loss & $9,601.03$ & 66.5 & -879.4 \\
Death & $\$ 1,185,354,128$ & $10,480.47$ & $-\$ 132,823,511$ \\
Annual direct medical costs & & $\$ 1,318,177,638$ & PCV13 dominates PCV10 \\
\hline Cost per QALY & & & \\
\hline
\end{tabular}

PCV10 10-valent pneumococcal conjugate vaccine; PCV13 13-valent pneumococcal conjugate vaccine; QALY quality-adjusted life-year. 
Table 5 Scenario analysis cost and outcomes when considering direct effects only

\begin{tabular}{llll}
\hline Outcome & PCV13 & PCV10 & Difference (PCV13 - PCV10) \\
\hline Total life-years & $605,587,157$ & $605,586,870$ & 287.45 \\
Total QALYs & $543,278,132$ & $543,264,303$ & 257.70 \\
\hline Annual number of cases & 1,906 & & -11.0 \\
Myringotomy & 35.7 & 1,918 & -0.1 \\
Neurological impairment & 66.3 & 35.8 & -0.2 \\
Hearing loss & $10,466.50$ & 66.5 & -14.0 \\
Death & $\$ 1,312,418,621$ & $10,480.47$ & $-\$ 5,759,017$ \\
Annual direct medical costs & & $\$ 1,318,177,638$ & PCV13 dominates PCV10 \\
\hline Cost per QALY & &
\end{tabular}

PCV10 10-valent pneumococcal conjugate vaccine; PCV13 13-valent pneumococcal conjugate vaccine; QALY quality-adjusted life-year.

\section{Discussion}

We developed a decision-analytic model to compare the costs and outcomes associated with a national immunization program of PCV13, as is currently implemented throughout Canada, compared with PCV10, which was previously used in some provinces following usage of PCV7 and before transitioning to PCV13. Overall, widespread use of PCV13 in Canada was expected to have a substantial public-health impact and be cost-saving due to its demonstrated immunogenicity and broader serotype coverage when compared with PCV10.

Our analysis approach was similar to other Canadianspecific analyses in the literature, in terms of the decisionanalytic model structure, diseases considered, and approaches to input data $[13,14,25,31]$. Talbird et al. performed an all-Canada analysis that compared the use of PCV10 to PCV7. Thus, it was difficult compare results of our analysis to that analysis. Although we saw many similarities in the input data (i.e., incidence, mortality, costs, sequelae utilities), Talbird and colleagues drew their serotype coverage data from a different surveillance-coordinating center and estimated serotype coverage as an average over

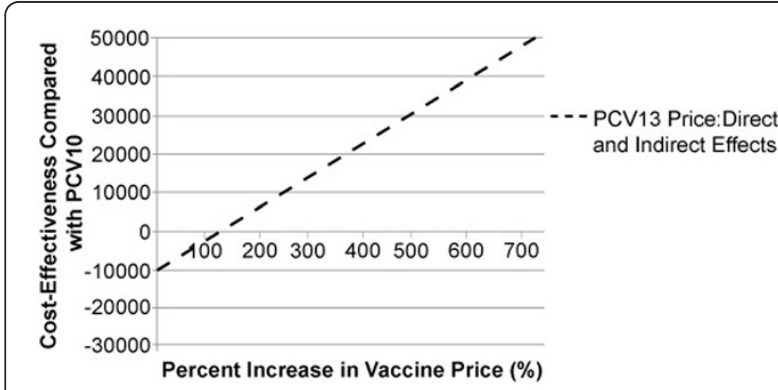

Figure 2 Breakeven Analysis. PCV10 = 10-valent pneumococcal conjugate vaccine; PCV13 = 13-valent pneumococcal conjugate vaccine. Dashed line represents the percentage increase in PCV13 cost per dose over PCV10 cost per dose that could occur at various cost-effectiveness thresholds when direct effects and indirect effects are considered. a 10-year period. Conversely, we considered current serotype coverage as reported by the National Centre for Streptococcus. In addition, Talbird et al. considered PCV10 direct effects on NTHi in IPD and AOM, in which the effect of PCV10 on NTHi-caused AOM was substantial $(30 \%-38 \%$ of AOM due to Streptococcus pneumonia and $38 \%-57 \%$ of non-Streptococcus pneumonia disease due to NTHi) [31]. Talbird et al. also considered a utility decrement due to having AOM, making the approach in our analysis more conservative [55].

Chuck et al. [14] compared the use of PCV10 to PCV13 in Alberta. Their results differed slightly in that they found PCV10 to be cost-effective when considering coverage of NTHi in AOM. Although our analyses were similar around assumptions on utilities, incidence of disease sequelae, and costs, Chuck et al. assumed a greater rate of mortality. In addition, Chuck and colleagues considered an incidence combined with serotype coverage in Alberta as estimated from the National Centre for Streptococcus. An in-depth comparison of the incidence and serotype coverage data values found that the incidence along with serotype coverage assumed in their analysis was much lower than assumed in our analysis. A comparison of these data values with Talbird et al. showed our data values to be more in line with Talbird et al. [31]. It is unclear whether the incidence along with serotype coverage was much lower in Alberta than for the rest of Canada. As a result, the value of PCV13 could be overestimated. All in all, to account for these differences, we performed multiple scenario analyses around our assumptions on both incidence and serotype coverage. It will be important for decision makers to examine these analyses closely and determine which analysis most closely resembles their situation.

The disease epidemiology has changed dramatically since the introduction of PCV7 in 2003 [1-8]. Specifically, there has been a fair amount of serotype replacement. Figure 4 shows the trends of PCV7-covered serotypes over time, as measured by a number of 


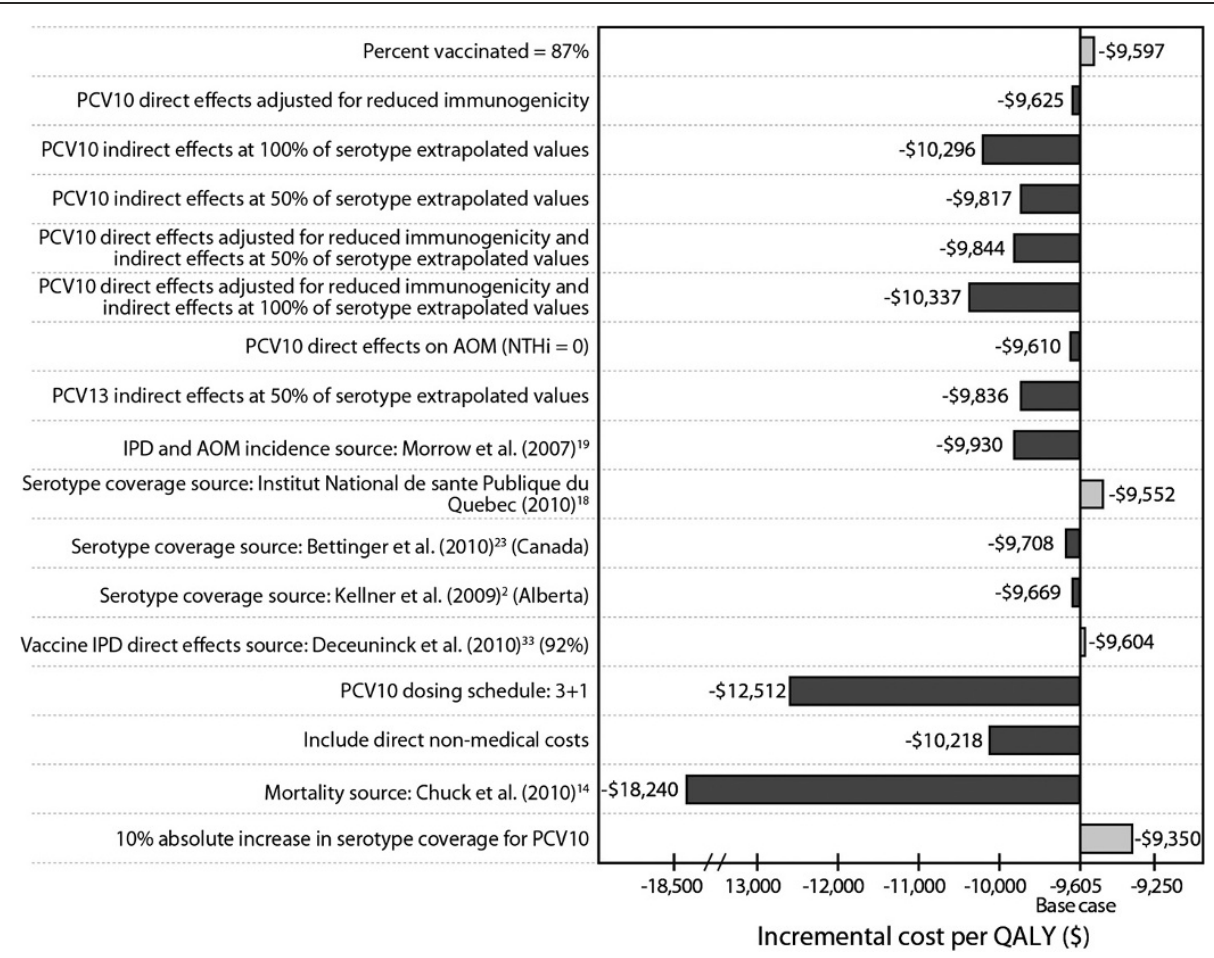

Figure 3 Sensitivity analysis results (Based on a base case of direct and indirect effects being assumed). AOM=acute otitis media; IPD = invasive pneumococcal disease; NTHi = non-typeable Haemophilus influenzae; PCV10 = 10-valent pneumococcal conjugate vaccine; PCV13 = 13-valent pneumococcal conjugate vaccine; QALY = quality-adjusted life-year. Dark shaded bar represents an increase in value for the denoted parameter. Light shaded bar represents a decreased in value for the denoted parameter.

surveillance studies. As seen in Figure 4a, while PCV7covered serotypes have decreased approximately 6-fold, PCV7 non-covered serotypes have increased 2- to 4-fold in various Canadian territories and provinces. From the Institut National de Sante Publique du Quebec, we also observed that in the early years of PCV7, PCV7 covered approximately $50 \%$ of pneumococcal disease (Figure $4 \mathrm{~b}$ ) [18]. Over the years and because of the implementation of PCV7, PCV7-covered serotypes have been dramatically reduced. Examining current serotype coverage, we observed that PCV13 was estimated to cover approximately $50 \%$ of pneumococcal disease. In addition, data from Laboratoire De La Sante Publique de Quebec for 2009 showed 19A as the most prevalent serotype across all ages, covering nearly $18 \%$ of all serotype isolates in Quebec and representing almost $68 \%$ of vaccine-preventable IPD covered by PCV 13. Data from the National Centre for Streptococcus for 2009-2010 confirmed 19A to be the most prevalent among 0- to 4-year-olds [23]. With PCV13 being the only vaccine to cover 19A, significant reductions in disease might be expected. Sensitivity analysis using the various serotype coverage estimates showed PCV13 to remain cost-saving when compared with PCV10.

PCV10 has been shown to have a lower immunogenic response than PCV7 among the seven common serotypes, as demonstrated by lower geometric mean antibody concentrations and by percentage of infants achieving serum immunoglobulin G seropositivity $\geq 0.20 \mu \mathrm{g} / \mathrm{mL}$ [10]. In our base-case analysis, we did not consider the lower immunogenic effect of PCV10. However, in sensitivity analysis we examined the impact of reduced effectiveness and showed that PCV13 continued to dominate PCV10. PCV7 consistently demonstrated a statistically significant reduction in nasopharyngeal carriage $[45,56,57]$, which is a critical component to creating an indirect effect. The data on statistically significant reduction in nasopharyngeal carriage is now emerging [48]. PCV10 has not demonstrated this reduction [10,51]. As a result, we assumed no indirect effects to PCV10 in the base case. It is possible that PCV10 could exhibit indirect effects. Thus, we examined the presence and absence of indirect effects for PCV10 and PCV13, respectively, in sensitivity analyses. In all analyses, PCV13 was found to remain cost-saving compared with PCV10.

In cost-effectiveness analyses of pneumococcal conjugate vaccines, assumptions around $\mathrm{AOM}$ incidence and efficacy played an important role in the direct medical costs accrued. Although the costs associated with a single AOM event were low relative to hospitalized disease, due to the high incidence of AOM these costs were a 


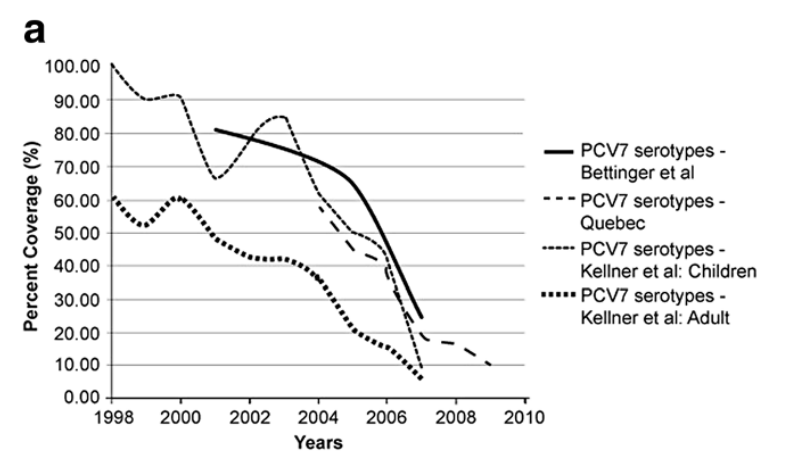

b

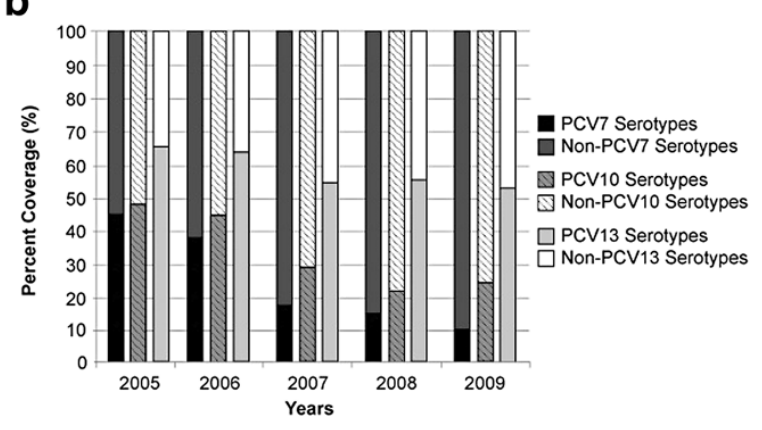

Figure $\mathbf{4}$ Change in PCV7 and non-PCV7 serotype coverage over time. (a) Change in PCV7 Serotype Coverage Over Time. PCV7 = 7-valent pneumococcal polysaccharide-protein conjugate vaccine. Solid line represents coverage of PCV7 serotypes for Canada as reported by Bettinger et al. (2010). Dashed line represents coverage of PCV7 serotypes for Quebec the Institut National de Sante Publique du Quebec (2010). Dashed-dotted line represents coverage of PCV7 serotypes for children in Alberta as reported by Kellner et al. (2009). Square-dotted line represents coverage of PCV7 serotypes for adults in Alberta as reported by Kellner et al. (2009). (b) Serotype Coverage for All Pneumococcal Vaccines Over Time. PCV7 = 7-valent pneumococcal polysaccharide-protein conjugate vaccine; $P C V 10=10$-valent pneumococcal conjugate vaccine; PCV13 = 13-valent pneumococcal conjugate vaccine. Dark solid bar represents PCV7 serotype. Medium solid bar represents non-PCV7 serotypes. Dark striped bar represents PCV10 serotypes. Light striped bar represents non-PCV10 serotypes. White-shaded bar represents PCV13 serotypes. Light-shaded bar represents non-PCV13 serotypes.

substantial contributor to the disease burden calculated within our model. In addition, because the etiology of AOM is typically all-cause, a critical component to the cost-effectiveness of pneumococcal vaccines was the impact of vaccine effectiveness on NTHi. Recent economic analyses have shown that PCV10 reduces costs and is cost-effective when effectiveness on NTHi is considered $[14,58]$. Chuck et al. [14] assumed that PCV10 would have a $3 \%$ increase in direct effects on all AOM due to NTHi. However, Talbird et al. presented an etiologyweighted estimate of $22.9 \%$ reduction in all-cause AOM relative to $6.7 \%$ for PCV7. Subsequent analyses also used this approach [58,59]. Details of this approach have been discussed elsewhere $[55,60]$.
In this analysis, we considered both direct effects and a combination of direct and indirect effects of the vaccines. However, evidence of indirect effects for PCV10 and PCV13 are limited in the literature. Indirect effects for PCV7 have been firmly established in the Canada, the US, Australia, and the UK [2,24,45,46,61-63]. Because PCV13 shares the same carrier protein as PCV7, we assumed PCV13 would incur similar indirect effects as PCV7. In addition, a recent analysis performed by the CDC demonstrated a decline in PCV13-type IPD in adults in the US [49]. However, we acknowledge that a recent presentation by De Wals and colleagues [64] noted that the existence of indirect effects in the presence of PCV7 for all-cause PNE could not be identified in Quebec. An indirect effect for IPD in adults was observed, but the authors also observed "strong and rapid" serotype replacement. Thus, a targeted vaccination program in adults may provide better protection to this group [64]. We examined of the use of PCV10 and PCV13 in the presence and absence of indirect effects and found PCV13 to be cost-saving in both scenarios. We acknowledge that we did not explicitly examine the impact of serotype replacement following the use of higher-valent conjugate vaccines. As these data become available, it will be important to consider this impact.

A limitation of this model is the uncertainty around vaccine prices due to the confidential nature of tender negotiations. When including indirect effects, our model was not sensitive to PCV13 price variation; PCV13 remained a cost-saving option even if PCV13 was twice the price of PCV10. In addition, PCV13 remained cost-saving regardless of the reduction in cost per dose for PCV10 when the cost per dose of PCV13 was held constant. This was due to the substantial burden of disease caused by PCV13-specific serotypes compared with PCV10-covered serotypes. This difference in coverage represents more than $27 \%$ of all serotypes in Quebec [18]. Due to the confidential nature of negotiated tenders, it will be important for decision-makers to perform these analyses under their own pricing scenarios so that they can better understand the economic value of PCV13 compared with PCV10.

\section{Conclusions}

In conclusion, our analysis shows PCV13 to be cost-saving compared with PCV10, given the most recently available epidemiology of disease and the potential effects of PCV10 and PCV13. Because of the burden of disease that is caused by serotype 19A, provinces and territories have adopted the use of PCV13. Further research will be needed to monitor the effect of PCV13 on disease in Canada, including the effect on 19A in vaccinated and unvaccinated populations and on emergent non-vaccine serotypes. However, health directors in provinces and territories should be assured that they have implemented a cost-savings policy. 


\section{Additional file}

Additional file 1: Table A-1. Sensitivity Analysis Input Parameters.

\author{
Abbreviation \\ AOM: acute otitis media; IPD: invasive pneumococcal disease; NTHi: non-typeable \\ Haemophilus influenza; PCV7: 7-valent pneumococcal polysaccharide-protein \\ conjugate vaccine; PCV10: 10-valent pneumococcal conjugate vaccine; \\ PCV13: 13-valent pneumococcal conjugate vaccine; PNE: pneumonia; \\ QALY: quality-adjusted life-year.
}

\section{Competing interests}

This study was sponsored by Pfizer Canada, Inc. Giovanni Zanotti is an employee of Pfizer Canada, Inc., and Raymond A. Farkouh is an employee of Pfizer Inc., which manufacturers 7-valent and 13-valent pneumococcal polysaccharideprotein conjugate vaccines. Stephanie R. Earnshaw and Cheryl L. McDade are employees of RTI Health Solutions, an independent contract research organization that has received research funding from Pfizer Canada, Inc. for this and other research studies, as well as funding from other pharmaceutical companies that market vaccines and drugs for other medical conditions.

\section{Acknowledgments}

This manuscript was funded by Pfizer Canada, Inc.

\section{Author details}

${ }^{1}$ RTI Health Solutions, 200 Park Offices Drive, Research Triangle Park, NC 27709, USA. 2Pfizer Canada Inc., 17300 Trans Canada, Kirkland, Quebec H9J 2M5, Canada. ${ }^{3}$ Pfizer Inc., 500 Arcola Road, Collegeville, PA 19426, USA.

\section{Authors' contributions}

SRE conceptualised the design of the study, acquired data, performed analysis, and interpreted data. She also drafted the manuscript, reviewed and revised the manuscript critically for important content, and approved the final manuscript. CM was involved in the conceptualisation of the design of the study, acquired data, programmed the decision model, performed the analysis, reviewed and revised the manuscript critically for important content, and approved the final manuscript. GZ was involved in acquisition of data, interpretation of data, review and revision of the manuscript critically for important content, and approved the final manuscript. RAF was involved in interpretation of data, review and revision of the manuscript critically for important content, and approved the final manuscript. DS was involved in the conceptualisation of the design of the study, interpretation of data, and approved the final manuscript.

Received: 11 October 2011 Accepted: 24 April 2012

Published: 24 April 2012

\section{References}

1. Kellner JD, Church DL, MacDonald J, Tyrrell GJ, Scheifele D: Progress in the prevention of pneumococcal infection. CMAJ 2005, 173(10):1149-1151.

2. Kellner JD, Vanderkooi OG, MacDonald J, Church DL, Tyrrell GJ, Scheifele DW: Changing epidemiology of invasive pneumococcal disease in Canada, 1998-2007: update from the Calgary-area Streptococcus pneumoniae research (CASPER) study. Clin Infect Dis 2009, 49(2):205-212.

3. Kellner JD, Scheifele D, Vanderkooi OG, MacDonald J, Church DL, Tyrrell GJ: Effects of routine infant vaccination with the 7-valent pneumococcal conjugate vaccine on nasopharyngeal colonization with Streptococcus pneumoniae in children in Calgary, Canada. Pediatr Infect Dis J 2008, 27(6):526-532

4. Tyrrell GJ, Lovgren M, Chui N, Minion J, Garg S, Kellner JD, Marrie TJ: Serotypes and antimicrobial susceptibilities of invasive Streptococcus pneumoniae pre- and post-seven valent pneumococcal conjugate vaccine introduction in Alberta, Canada, 2000-2006. Vaccine 2009, 27(27):3553-3560.

5. Bjornson G, Scheifele DW, Bettinger J, Patrick DM, Gustafson L, Daly P, Tyrrell GJ: Effectiveness of pneumococcal conjugate vaccine in Greater Vancouver, Canada: 2004-2005. Pediatr Infect Dis J 2007, 26(6):540-542.

6. Paulus S, David ST, Tang W, Winters M, Buxton J, Henry B, Patrick D: Incidence of invasive pneumococcal disease after introduction of the Universal Infant Immunization Program, British Columbia (2002-2005). Can Commun Dis Rep 2006, 32(14):157-161.
7. Degani N, Navarro C, Deeks SL, Lovgren M: Invasive bacterial diseases in northern Canada. Emerg Infect Dis 2008, 14(1):34-40.

8. Langley JM, Kellner JD, Solomon N, Robinson JL, Le Saux N, McDonald J, Ulloa-Gutierrez R, Tan B, Allen U, Dobson S, Joudrey H: Empyema associated with community-acquired pneumonia: a Pediatric Investigator's Collaborative Network on Infections in Canada (PICNIC) study. BMC Infect Dis 2008, 8:129.

9. Synflorix Product Monograph. Pneumococcal conjugate vaccine (non-typeable Haemophilus influenzae [NTHi] protein D, diphtheria or tetanus toxoid conjugates) adsorbed. Mississauga, Ontario, Canada: GlaxoSmithKline, Inc; May 5, 2009.

10. Prymula R, Peeters P, Chrobok V, Kriz P, Novakova E, Kaliskova E, Kohl I, Lommel P, Poolman J, Prieels JP, Schuerman L: Pneumococcal capsular polysaccharides conjugated to protein $D$ for prevention of acute otitis media caused by both Streptococcus pneumoniae and non-typeable Haemophilus influenzae: a randomised double-blind efficacy study. Lancet 2006, 367(9512):740-748.

11. Prevnar*13 Product Monograph. Pneumococcal 13-valent conjugate vaccine (diphtheria CRM197 protein). Kirkland, Quebec, Canada: Pfize Canada, Inc; November 16, 2010.

12. Synflorix Summary of Product Characteristics. Pneumococcal conjugate vaccine (non-typeable Haemophilus influenzae [NTHi] protein $\mathrm{D}$, diphtheria or tetanus toxoid conjugates) adsorbed. Rixensart, Belgium: GlaxoSmithKline Biologicals SA; January 24, 2011. [http:// www.medicines.org.uk/EMC/medicine/22743/SPC/Synflorix+suspension+for +injection+in+pre-filled+syringe/\#FORM]. Accessed February 28, 2011.

13. Talbird SE, Taylor TN, Knoll S, Fostad CR, Marti SG: Outcomes and costs associated with PHiD-CV, a new protein D conjugate pneumococcal vaccine, in four countries. Vaccine 2010, 28S:G23-G29.

14. Chuck AW, Jacobs P, Tyrrell G, Kellner JD: Pharmacoeconomic evaluation of 10- and 13-valent pneumococcal conjugate vaccines. Vaccine 2010, 28:5485-5490.

15. Roussy JP, Kwan H, Earnshaw SR, Farkouh RA, Hwang S, Strutton D. Cost-effectiveness of 10-valent pneumococcal conjugate vaccine compared with 10-valent vaccine in preventing pneumococcal infection in Quebec. Poster presented at the 51st Interscience Conference on Antimicrobial Agents and Chemotherapy; Boston, MA. September 12-15, 2010.

16. Comite sur L'Immunisation du Quebec: Evaluation of two new pneumococcal conjugate vaccines for pediatric immunization in Quebec. Quebec: Institute National de Sante Publique du Quebec; October 2010.

17. Statistics Canada. CANSIM, table (for fee) 051-0001. Population by sex and age group. 2010a Canada. [http://www40.statcan.gc.ca/l01/cst01/demo10aeng.htm. Accessed February 28, 2011].

18. Institut National de Sante Publique du Quebec: Programme de surveillance due pneumocoque: rapport 2009. Quebec: Laboratoire de santé publique du Quebec; September 2010.

19. Morrow A, De Wals P, Petit G, Guay M, Erickson L: The burden of pneumococcal disease in Canadian population before routine use of the seven-valent pneumococcal conjugate vaccine. Can J Infect Dis Med Microbiol 2007, 18(2):121-127.

20. Petit G, De Wals P, Law B, Tam T, Erickson L, Guay M, Framarin A: Epidemiological and economic burden of pneumococcal diseases in Canadian children. Can J Infect Dis 2003, 14(4):215-220.

21. Nelson JC, Jackson M, Yu O, Whitney CG, Bounds L, Bittner R, Zavitkorsky A, Jackson LA: Impact of the introduction of pneumococcal conjugate vaccine on rates of community acquired pneumonia in children and adults. Vaccine 2008, 26:4947-4954.

22. De Wals P, Carbon M, Sevin E, Deceuninck G, Ouakki M: Reduced physician claims of otitis media after implementation of pneumococcal conjugate vaccine program in the province of Quebec, Canada. Pediatr Infect Dis J 2009, 28(9):e271-e274.

23. Giltner CL., Lovgren M, McGeer A, Hoang L, Horsman GB, Van Caseele P,Tyrrell GJ. Serotype distribution of Streptococcus pneumoniae in Canada from 1991 to 2009. Poster presented at 2011 PDF Research Day conference; University of Alberta, Edmonton, Alberta, Canada. May 11, 2011.

24. Bettinger JA, Scheifele DW, Kellner JD, Halperin SA, Vaudry W, Law B, Tyrrell $G$, for members of the Canadian Immunization Monitoring Program, Active (IMPACT): The effect of routine vaccination on invasive pneumococcal infection in Canadian children, immunization monitoring program, active 2000-2007. Vaccine 2010, 28:2130-2136. 
25. Talbird SE, Ismaila AS, Taylor TN: A steady-state, population-based model to estimate the direct and indirect effects of pneumococcal vaccines. Vaccine 2010, 28S:G3-G13.

26. Statistics Canada. Health trends. 2010b. Statistics Canada catalogue no. 82-213-XWE. [http://www12.statcan.gc.ca/health-sante/82-213/index.cfm? Lang=ENG]. Accessed March 2, 2011.

27. Scheifele D, Halperin S, Pelletier L, Talbot J, members of the Canadian Paediatric Society/Laboratory Centre for Disease Control Immunization Monitoring Program, Active (IMPCAT): Invasive pneumococcal infections in Canadian children, 1991-1998: implications for new vaccination strategies. Clin Infect Dis 2000, 31:58-64.

28. Jette LP, Delage G, Ringuette L, Allard R, De Wals P, Lamothe F, Loo V, Pneumococcus Study Group: Surveillance of invasive Streptococcus pneumoniae infection in the province of Quebec, Canada, from 1996 to 1998: Serotype distribution, antimicrobial susceptibility, and clinical characteristics. J Clin Microbiol 2001, 733:737.

29. Kaye PA, Andrews NJ, Slack MPE, George RC, Miller E. The impact of pneumococcal conjugate vaccination in children in England and Wales four years after the introduction of PCV7. Poster presented at the European Society of Pediatric Infectious Diseases; Den Haag, Netherlands. June 7-11, 2011. Abstract No. 713.

30. Tregnaghi MW, Sáez-Llorens X, López P, Abate H, Smith E, Pósleman A, Calvo A, Wong D, Cortes-Barbosa C, Ceballos A, Tregnaghi M, Sierra $A$, Márquez $V$, Rodriguez $M$, Troitiño $M$, Rüttimann $R$, Castrejón MM, Lepetic A, Lommel P, Hausdorff WP, Borys D, Ruiz Guiñazú J, Ortega-Barría E, Yarzábal JP, Schuerman L. Evaluating the efficacy of 10-valent pneumococcal non-typeable haemophilus influenza protein-D conjugate vaccine (PHid-CV) against community-acquired pneumonia in Latin America. Poster presented at the European Society of Pediatric Infectious Diseases; Den Haag, Netherlands. June 7-11 2011. Abstract No. 412

31. Talbird SE, Taylor TN, Caporale J, Ismaila AS, Gomez J: Residual economic burden of Streptococcus pneumonia- and nontypeable Haemophilus influenza-associated disease following vaccination with PCV-7: a multicountry analysis. Vaccine 2010, 28S:G14-G22.

32. Rubin JL, McGarry LJ, Strutton DR, Klugman KP, Pelton SI, Gilmore KE, Weinstein MC: Public health and economic impact of the 13-valent pneumococcal conjugate vaccine (PCV13) in the United States. Vaccine 2010, 28(48):7634-7643.

33. Black S, Shinefield H, Baxter R, Austrian R, Elvin L, Hansen J, Lewis E, Fireman B: Impact of the use of heptavalent pneumococcal conjugate vaccine on disease epidemiology in children and adults. Vaccine 2006, 24(suppl 2):S79-\$80.

34. Deceuninck G, De Wals P, Boulianne N, De Serres G: Effectiveness of pneumococcal conjugate vaccine using a $2+1$ infant schedule in Quebec, Canada. Pediatr Infect Dis J 2010, 29(6):546-549.

35. Vesikari T, Wysocki J, Chevallier B, Karvonen A, Czajka H, Arsène JP, Lommel $P$, Dieussaert I, Schuerman L: Immunogenicity of the 10-valent pneumococcal non-typeable Haemophilus influenzae protein D conjugate vaccine (PhiD-CV) compared to the licensed 7vCRM vaccine. Pediatr Infect Dis J 2009, 28:S66-S76.

36. Goldblatt D. Establishing the correlate for pneumonia. Podium presentation at the 6th International Symposium on Pneumococci and Pneumococcal Diseases; Reykjavik, Iceland. June 11, 2008.

37. Yeh SH, Gurtman A, Hurley DC, Block SL, Schwartz RH, Patterson S, Jansen KU, Love J, Gruber WC, Emini EA, Scott DA, 004 Study Group: Immunogenicity and safety of 13 -valent pneumococcal conjugate vaccine in infants and toddlers. Pediatrics 2010, 126(3):e493-e505. E-pub 2010 Aug 23.

38. Esposito S, Tansey S, Thompson A, Razmpour A, Liang J, Jones TR, Ferrera G, Maida A, Bona G, Sabatini C, Pugni L, Emini EA, Gruber WC, Scott DA, Principi N: Safety and immunogenicity of a 13-valent pneumococcal conjugate vaccine compared to those of a 7-valent pneumococcal conjugate vaccine given as a three-dose series with routine vaccines in healthy infants and toddlers. Clin Vaccine Immunol 2010, 17(6):1017-1026. E-pub 2010 Apr 28.

39. Bryant KA, Block SL, Baker SA, Gruber WC, Scott DA, for the PCV13 Infant Study Group: Safety and immunogenicity of a 13-Valent pneumococcal conjugate vaccine. Pediatrics 2010, 125:866. doi:10.1542/peds.2009-1405.

40. Ray GT, Whitney CG, Fireman BH, Ciuryla V, Black SB: Cost-effectiveness of pneumococcal conjugate vaccine: evidence from the first five years of use in the United States incorporating herd effects. Pediatr Infect Dis J 2006, 25:6.

41. Black SB, Shinefield HR, Ling S, Hansen J, Fireman B, Spring D, Noyes J, Lewis E, Ray P, Lee J, Hackell J: Effectiveness of heptavalent pneumococcal conjugate vaccine in children younger than five years of age for prevention of pneumonia. Pediatr Infect Dis J 2002, 21:810-815.

42. Fireman B, Black SB, Shinefield HR, Lee J, Lewis E, Ray P: Impact of the pneumococcal conjugate vaccine on otitis media. Pediatr Infect Dis J 2003, 22:10-16.

43. van Gils EJ, Veenhoven $R H$, Hak E, Rodenburg GD, Bogaert D, ljzerman EP, Bruin JP, van Alphen L, Sanders EA: Effect of reduced-dose schedules with 7-valent pneumococcal conjugate vaccine on nasopharyngeal pneumococcal carriage in children. A randomized controlled trial. JAMA 2009, 302(2):159-167.

44. Zhou F, Kyaw MH, Shefer A, Winston CA, Nuorti JP: Health care utilization for pneumonia in young children after routine pneumococcal conjugate vaccine use in the United States. Arch Pediatr Adolesc Med 2007, 161:1162-1168.

45. Pilishvili T, Lexau C, Farley MM, Hadler J, Harrison LH, Bennett NM, Reingold A, Thomas A, Schaffner W, Craig AS, Smith PJ, Beall BW, Whitney CG, Moore MR: Active Bacterial Core Surveillance/Emerging Infections Program Network. Sustained reductions in invasive pneumococcal disease in the era of conjugate vaccine. $J$ Infect Dis 2010, 201(1):32-41.

46. Miller E, Andrews NJ, Waight PA, Slack MP, George RC: Herd immunity and serotype replacement 4 years after seven-valent pneumococcal conjugate vaccination in England and Wales: an observational cohort study. Lancet Infect Dis 2011, 11(10):760-768. Epub 2011 May 27.

47. Koshy E, Murray J, Bottle A, Sharland M, Saxena S: Impact of the seven-valent pneumococcal conjugate vaccination (PCV7) programme on childhood hospital admissions for bacterial pneumonia and empyema in England: national time-trends study, 1997-2008. Thorax 2010, 65(9):770-774.

48. Cohen R, Levy C, Bingen E, Bonnet E, Koskas M, Attal S, Nave I, Fritzell B, Varon E. Impact of 13-valent pneumococcal conjugate vaccine (PCV13) on nasopharyngeal (NP) flora in children with acute otitis media (AOM). Podium presentation at the 51st Interscience Conference on Antimicrobial Agents and Chemotherapy; Chicago, IL. September 17-20, 2011

49. Moore M, Link-Gelles R, Farley M, Schaffner W, Thomas A, Reingold A, Harrison L, Lexau C, Zansky S, Petit S, Gershman K, Scherzinger K, Juni B, Beall B, Whitney C. Impact of 13-valent pneumococcal conjugate vaccine on invasive pneumococcal disease, United States, 2010-11. Presented at 8th International Symposium on Pneumococci \& Pneumococcal Diseases. March 13, 2012.

50. Prymula R, Kriz P, Kaliskova E, Pascal T, Poolman J, Schuerman L: Effect of vaccination with pneumococcal capsular polysaccharides conjugated to Haemophilus influenzae-derived protein D on nasopharyngeal carriage of Streptococcus pneumoniae and $\mathrm{H}$. influenzae in children under 2 years of age. Vaccine 2009, 28(1):71-78.

51. Prymula R, Hanovcova I, Splino M, Kriz P, Motlova J, Lebedova V, Lommel P, Kaliskova E, Pascal T, Borys D, Schuerman L: Impact of the 10-valent pneumococcal non-typeable Haemophilus influenzae Protein D conjugate vaccine (PHiD-CV) on bacterial nasopharyngeal carriage. Vaccine 2011, 29(10):1959-1967

52. Statistics Canada. CANSIM table (for fee) 326-0021 and catalogue nos. 62-001-X, 62-010-X, and 62-557-X. Consumer Price Index, historical summary (1991-2010). 2011. [http://www40.statcan.ca/I01/cst01/ econ46a-eng.htm]. Accessed February 28, 2011.

53. De Wals P, Petit G, Erickson LJ, Guay M, Tam T, Law B, Framarin A: Benefits and costs of immunization of children with pneumococcal vaccine in Canada. Vaccine 2003, 21:3757-3764.

54. Statistics Canada. Canadian Community Health Survey. Cycle 2.1. 2003. [http://www.statcan.ca/english/concepts/health/cycle2_1/index.htm]. Accessed February 28, 2011

55. Talbird S, Knerer G, Hausdorff W, Taylor T, Frostad CR. Reply to Strutton et al:: Response to "Outcomes and costs associated with PHiD-CV, a new protein D conjugate pneumococcal vaccine, in four countries." Vaccine 2011, in press.

56. Dagan R, Givon-Lavi N, Zamir O, Fraser D: Effect of a nonvalent conjugate vaccine on carriage of antibiotic-resistant Streptococcus pneumoniae in day-care centers. Pediatr Infect Dis J 2003, 22(6):532-540. 
57. Huang SS, Hinrichsen VL, Stevenson AE, Rifas-Shiman SL, Kleinmand K, Pelton SI, Lipstich M, Hanage WP, Lee GM, Finkelstein JA: Continued impact of pneumococcal conjugate vaccine on carriage in young children. Pediatrics 2009, 124(1):e1-e11.

58. Urueña A, Pippo T, Betelu MS, Virgilio F, Giglio N, Gentile A, Jimenez SG, Jáuregui B, Clark AD, Diosque M, Vizzotti C: Cost-effectiveness analysis of the 10- and 13-valent pneumococcal conjugate vaccines in Argentina. Vaccine 2011, 29(31):4963-4972. E-pub 2011 May 27.

59. Morano R, Pérez F, Brosa M, Escolano IP. Cost-effectiveness analysis of pneumococcal vaccination in Spain. Gac Sanit 2011 Jun 1, in press.

60. Strutton D, Hwang S, Farkouh R, Roberts C. Response to "Outcomes and costs associated with PHiD-CV, a new protein D conjugate pneumococcal vaccine, in four countries." Vaccine 2011, in press.

61. Grijalva CG, Nuorti JP, Arbogast PG, Martin SW, Edwards KM, Griffin MR: Decline in pneumonia admissions after routine childhood immunization with pneumococcal conjugate vaccine in the USA: a time-series analysis. Lancet 2007, 369:1179-1186.

62. Roche PW, Krause V, Cook H, Barralet J, Coleman D, Sweeny A, Fielding J, Giele C, Gilmour R, Holland R, Kampen R, Enhanced Invasive Pneumococcal Disease Surveillance Working Group, Brown M, Gilbert L, Hogg G, Murphy D, Pneumococcal Working Party of the Communicable Diseases Network Australia: Invasive pneumococcal disease in Australia, 2006. Commun Dis Intell 2008, 32(1):18-30

63. Lehmann D, Willis J, Moore HC, Giele C, Murphy D, Keil Ad, Harrison C, Bayley K, Watson M, Richmond P: The changing epidemiology of invasive pneumococcal disease in aboriginal and non-aboriginal western Australians from 1997 through 2007 and emergence of nonvaccine serotypes. Clin Infect Dis 2010 Jun 1, 50(11):1477-1486.

64. De Wals P, Carbon M, Fortin E, Pepin J, Douville-Fradet M. Vaccination of children with pneumococcal conjugate vaccine had no impact on hospital admissions for pneumonia in adults in Quebec. Poster presented at the 51st Interscience Conference on Antimicrobial Agents and Chemotherapy; Boston, MA. September 12-15, 2010.

65. Hansen J, Black S, Shinefield H, Cherian T, Benson J, Fireman B, Lewis E, Ray $P$, Lee J: Effectiveness of heptavalent pneumococcal conjugate vaccine in children younger than 5 years of age for prevention of pneumonia: updated analysis using World Health Organization standardized interpretation of chest radiographs. Pediatr Infect Dis J 2006, 25:779-781.

doi:10.1186/1471-2334-12-101

Cite this article as: Earnshaw et al: Cost-effectiveness of $2+1$ dosing of 13-valent and 10-valent pneumococcal conjugate vaccines in Canada. BMC Infectious Diseases 2012, 12:101.

\section{Submit your next manuscript to BioMed Central and take full advantage of:}

- Convenient online submission

- Thorough peer review

- No space constraints or color figure charges

- Immediate publication on acceptance

- Inclusion in PubMed, CAS, Scopus and Google Scholar

- Research which is freely available for redistribution 